\title{
P18: Analyse von Sturzereignisprotokollen in einer vollstationären Altenpflegeeinrichtung
}

\author{
Nadine Fleischer-Schlechtiger · Jana Möbius-Winkler • \\ Jörg Klewer
}

Online publiziert: 23. Oktober 2013

(C) Springer-Verlag Wien 2013

Einleitung/Fragestellung: Sturzereignisse gefährden ältere pflegebedürftige Menschen und physische, psychische sowie gesundheitsökonomische Folgen können mit erheblichen Einbußen an Lebensqualität einher gehen. Für die Betroffenen kann ein Sturz physische Folgen, wie Mortalität, hüftnahe Frakturen, Immobilität, und psychische Folgen, wie Angst und damit einhergehend Verlust des Vertrauens in die eigene Mobilität, bedeuten. Für Pflegeeinrichtungen können sich ökonomische Sturzfolgen wie haftungsrechtliche Konsequenzen, erhöhte Kosten und Imageschäden ergeben.

Zielstellung: Es sollten die Ursachen der Sturzereignisse, die Anwendungsqualität der Sturzereignisprotokolle und daraus resultierende Maßnahmen in einer vollstationären Altenpflegeinrichtung untersucht werden.

Methodik/Stichprobe: Die Untersuchung wurde in einer vollstationären Pflegeeinrichtung mit insgesamt 74 Bewohnern durchgeführt. Es wurden alle anhand von Sturzereignisprotokollen dokumentierten 108 Sturzereignisse des Jahres 2012 analysiert.
Ergebnisse: Der überwiegende Anteil der gestürzten Bewohner waren in Pflegestufe II (47) und Pflegestufe I (37) eingruppiert und es stürzten mehr Frauen als Männer. Die Mehrfachsturzrate zeigte, dass mehr als die Hälfte der Bewohner zweimal und mehr stürzten. Meist ereigneten sich die Stürze am Nachmittag und zu den Dienstübergaben, vorwiegend unbeobachtet in den Bewohnerzimmern. Eine Überprüfung der sturzprophylaktischen Maßnahmen nach den Stürzen erfolgte nicht bei jedem Bewohner.

Diskussion/Schlussfolgerungen: Insgesamt konnte aus den gewonnenen Informationen ein bewohnerbezogenes Risikoprofil abgeleitet werden, wenngleich Defizite in der Umsetzung der Vorgaben des Expertenstandards aufgezeigt wurden. Der stürzende Bewohner in der untersuchten Einrichtung war über 85 Jahre alt, vorwiegend weiblich und den Pflegestufen I und II zuzuordnen. Überwiegend waren die Bewohner als mobil einzuschätzen, nutzten die Hilfsmittel selbstständig und waren zu den Stürzen ohne Beaufsichtigung.
N. Fleischer-Schlechtiger · J. Möbius-Winkler Volkssolidarität Leipziger Land/ Muldental e. V., Leipzig,

Deutschland

J. Klewer

Fakultät Gesundheits- und Pflegewissenschaften,

Westsächsische Hochschule Zwickau, Zwickau, Deutschland 